\title{
Analysis of the elliptic-profile cylindrical reflector with a non-uniform resistivity using the complex source and dual-series approach: H-polarization case
}

\author{
Taner Oğuzer · Ayhan Altıntaş • Alexander I. Nosich
}

Received: 9 October 2012 / Accepted: 8 May 2013 / Published online: 17 May 2013

C) Springer Science+Business Media New York 2013

\begin{abstract}
An elliptic-profile reflector with varying resistivity is analyzed under the illumination by an H-polarized beam generated by a complex-source-point (CSP) feed. The emphasis is done on the focusing ability that is potentially important in the applications in the optical range related to the partially transparent mirrors. We formulate the corresponding electromagnetic boundary-value problem and derive a singular integral equation from the resistive-surface boundary conditions. This equation is treated with the aid of the regularization technique called Riemann Hilbert Problem approach, which inverts the stronger singular part analytically, and converted to an infinite-matrix equation of the Fredholm 2 nd kind. The resulting numerical algorithm has guaranteed convergence. This type of solution provides more accurate and faster results compared to the known method of moments. In the computations, a CSP feed is placed into a more distant geometrical focus of the elliptic reflector, and the near-field values at the closer focus are plotted and discussed. Various farfield radiation patterns including those for the non-uniform resistive variation on the reflector are also presented.
\end{abstract}

Keywords Electromagnetic scattering - Method of analytical regularization · Optical devices

\section{Introduction}

The scattering of electromagnetic waves by the partially transparent thin curved scatterers, made up of lossless and lossy dielectric materials or imperfect metals, occupies a remarkable

\footnotetext{
T. Oğuzer $(\varangle)$

Electrical and Electronics Engineering Department, Dokuz Eylul University, Buca,

35160 Izmir, Turkey

e-mail: taner.oguzer@deu.edu.tr
}

A. Altıntaş

Department of Electrical and Electronics Engineering, Bilkent University, 06800 Ankara, Turkey

A. I. Nosich

Institute of Radio-Physics and Electronics NASU, Kharkiv 61085, Ukraine 
place in the electromagnetic wave theory. This can be understood because of, at least, two circumstances. Firstly it can be said that the well-known microwave reflector antenna systems are sometimes realized using thin resistive films, which cannot be simulated as perfectly electrically conducting (PEC) surfaces. This calls for a modified formulation of the corresponding wave-scattering problem. Secondly the same problem can be thought as generated by the micro-mirrors design in the optical systems because at optical wavelengths the PEC conditions are not valid at all.

Here, parabolic-profile thin screens and reflectors are most frequent because of the collimation and focusing properties. Still hyperbolic and elliptic sub-reflectors are used as the primary-feed illuminated parts of the large Cassegrain and Gregorian dual-reflector antennas with parabolic main dishes (Scott 1990). In the optical applications, sometimes it is necessary to provide highly directional radiation of laser that can be achieved using the micro-lenses and micro-mirrors, which can have elliptic. Besides, the optical or terahertz-wave beam can be guided along a finite chain of elliptic reflectors or micro-mirrors. Reliable and economic numerical simulation of these structures can be performed using the electromagnetic boundary-value problem formulations with modified boundary conditions.

In the full-wave modeling of the electromagnetic systems from the microwaves to optical range, finite-difference time-domain (FDTD) method is the one of the oldest techniques. It needs huge number of unknowns due to the discretization of the all physical domain and it also has a disadvantage in failing to satisfy the far-field radiation condition explicitly. In terms of the accuracy, FDTD may have some deficiency even in application to some simple circular-shell structures (Hower et al. 1993). Well-known alternative way is to obtain singular integral equation (SIE) from the corresponding boundary condition on the reflector. In this approach, the radiation condition is satisfied automatically and the numbers of unknowns are reduced drastically. However some problems still remain and they are associated with mathematical convergence and accuracy. For instance, a simple and well-known way is the method of moments (MoM) with local and global basis and testing functions (Jenn et al. 1995; Barclay and Rusch 1991; Heldring et al. 2004). In the application of the MoM to solve a SIE of Cauchy type or higher, the convergence of the solution is not guaranteed and strongly depends on the implementation. As a result, non realistic computation time may occur. Generally it is known that the MoM can be applied to small and medium size reflectors (up to 10 wavelengths); the achievable accuracy is frequently only a few digits for the surface current function.

The mathematically exact solution of certain reflector problems can be derived by using the modified Wiener-Hopf technique (Mittra and Lee 1971). For instance, a 2D curved strip scattering was studied in Idemen and Büyükaksoy (1984). This technique is important because it enables one to obtain the asymptotic high frequency expressions for the scattering characteristics. These alternatives are known as the high frequency techniques such as geometrical optics (GO), physical optics (PO) and physical theory of diffraction (PTD) especially for the larger PEC reflectors (Hasselmann and Felsen 1982; Suedan and Jull 1991; Martinez-Burdalo et al. 1993). Lately the PO has been combined with the Gaussian beam field decomposition aimed at the faster analysis of larger reflectors (Anastassiu and Pathak 1995; Chou et al. 2003; Rieckmann 2002), however this is still an approximation of the actual solution.

In the numerical modeling of the above mentioned geometries with the aid of SIEs a remarkable alternative is the method of analytical regularization (MAR) (Nosich 1999). In MAR, the kernel of the SIE is separated into two parts, the more singular part (usually static) and the remainder. In the H-polarization scattering by a PEC screen, the SIE involves the second derivative of the 2-D Green's function and hence is a hyper-singular equation. Then, 
the choice of the global basis functions that are orthogonal eigenfunctions of the most singular part enables one to perform analytical inversion by using the Riemann Hilbert problem (RHP) technique. The remainder leads to the Fredholm second-kind matrix equation that provides a convergent numerical solution. This technique, combined with the dual-series equations, was presented in detail in Nosich (1993) for a PEC 2-D circular screen and then applied to the modeling of various 2-D circular and non-circular reflector antenna systems in free space and in more complicated near-field environments (Oğuzer et al. 1995; Yurchenko et al. 1999; Oğuzer 2001; Oğuzer and Altıntaş 2005, 2007; Boriskina et al. 2000; Oğuzer et al. 2001, 2004); here, the CSP field was adopted to simulate a feed. In either case, the Green's functions of the host media have logarithmic singularities, and therefore the SIE-MAR techniques exploit the same ideas when performing the partial inversion.

As for the imperfect 2-D reflectors, one can firstly mention about the PO approximate treatment of the problem. In Senior (1978), the resistive half plane illuminated by a plane wave and then in Umul (2007), a beam excitation case of the same geometry has been studied by using the Modified-PO technique. Later a 2-D impedance parabolic reflector fed by a line source was studied with the same technique (Umul 2008). As mentioned, imperfect-reflector problems can be efficiently analyzed with the SIE-MAR technique. In Nosich et al. (1996), a circular 2-D reflector with a uniform resistivity surface was studied, illuminated by the plane waves. In Nosich et al. (1997), the same geometry but with non-uniform resistivity was modeled under the CSP-beam illumination. In that study, a non-uniform resistivity was realized as an almost-PEC reflector edge-loaded with a linearly increasing resistivity region. This edge-loaded case reduces the diffraction effects that are caused by the sharp edges of the PEC reflector, especially at the penumbra region and back side lobes of the radiation patterns. Lately in Oğuzer et al. (2009), we have studied the 2-D scattering problem assuming a noncircular contour with uniformly and non-uniformly resistive (i.e. edge-loaded) surface. Our aim was to simulate a parabolic reflector fed by a directive beam and therefore we used the CSP field as the incident wave. In the mentioned study, however, only the E-polarization case was treated with SIE-MAR that meant the inversion of the logarithmic singularity using the discrete Fourier inversion procedure.

In the present study, we consider the alternative case of $\mathrm{H}$-polarization of the same problem as in Oğuzer et al. (2009). In this case, the SIE is a hyper-type one due to the derivatives of the Green's function in the kernel however non-zero resistivity is a simple perturbation of the PEC case. Therefore the same RHP as in the PEC case technique is used to invert the most singular part and reduce the problem to a Fredholm second-kind matrix equation, whose numerical solution guarantees convergence. Our derivations and final algorithm are valid for arbitrary smooth contour of reflector with non-uniform resistivity; however unlike (Oğuzer et al. 2009) we concentrate our numerical study on the elliptic-profile reflectors. In the optical frequency range, this study has applications in the design of micro-size metallic mirrors used in the pump-radiation focusers for the semiconductor lasers. Finite chains of elliptic reflectors can be also used to build low-loss beam waveguides met in the heating of plasma with millimeter waves in controlled nuclear-fusion machines (Thumm and Kasparek 2002), in the tomography of the same plasma with sub-millimeter waves, and in the front-end circuits of millimeter and far-infrared wave receivers of radio astronomy antennas (Goldsmith 1998).

In Sect. 2, we formulate the boundary-value problem and derive hyper-singular IE. Section 3 is concerned with analytical regularization of SIE and its conversion to the matrix equation. In Sect. 4, we derive radiation characteristics. Section 5 contains the numerical results obtained for various reflector surfaces and resistivity cases. The conclusions are formulated in Sect. 6. 


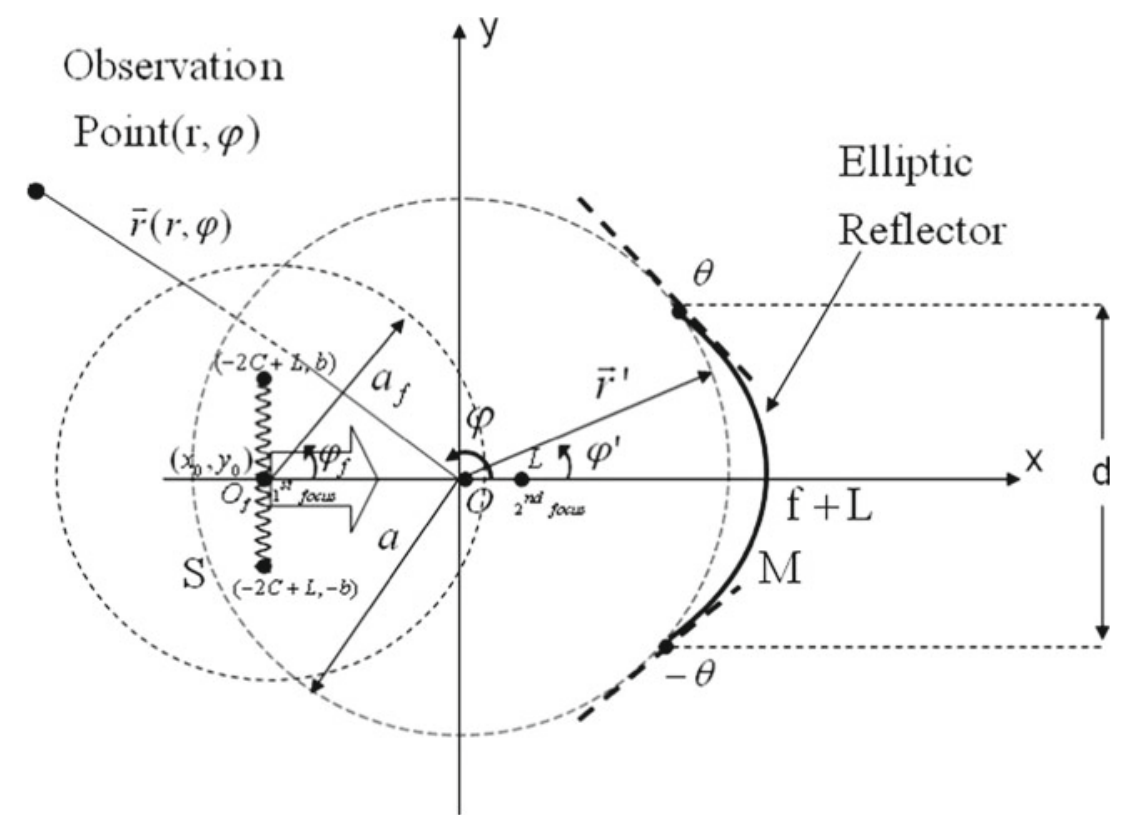

Fig. 1 The problem geometry for the finite elliptic-profile reflector. Thick dashed straight lines centered at reflector's edges mark the corresponding tangents. Zigzag line centered at (L, 0) marks the branch cut associated with the CSP source. $(\mathrm{c}=\mathrm{e} * \mathrm{f} /(1-\mathrm{e}), \mathrm{g}=\mathrm{f} /(1-\mathrm{e})$ where $\mathrm{f}=\mathrm{g}-\mathrm{c}$ and $\mathrm{e}$ is the eccentricity factor of ellipse. Also $\mathrm{c}$ and $\mathrm{g}$ are the widths of the ellipse in $\mathrm{x}$ and $\mathrm{y}$ directions)

\section{Formulation and sie}

The problem geometry associated with a very thin layer (compared to the wavelength) and the resistive front-fed reflector illuminated by a CSP feed is presented in Fig. 1. The 2-D cross section of the reflector is denoted as open contour $M$ and defined as conic-section profile having a symmetry line (the $x$ axis). It can be an elliptic, parabolic or hyperbolic arc, differing in the value of the parameter called eccentricity, $e$, so that $e<1$ for an ellipse; $e=1$ for a parabola and $e>1$ for a hyperbola. The virtual aperture open contour $S$ completes the open contour $M$ to the closed contour $C$ and is defined in such a way that $\mathrm{C}$ has continuous junction points (i.e. reflector's edge points). Additionally the arc $M$ should not cross the CSP branch-cut (see below); otherwise the resulting matrix equation looses convergence.

The total field in the presence of reflector is considered as a sum of the incident and scattered field,

$$
H_{z}^{T}=H_{z}^{i n}+H_{z}^{s c}
$$

The rigorous formulation of the considered boundary value problem is stated in terms of the Helmholtz equation, the Sommerfeld radiation condition far from the reflector and source, the resistive boundary condition on $M$, and the edge condition such that the totalfield power is limited in any finite domain including the reflector edge but excluding the source singularities. Collectively, these conditions guarantee the uniqueness of the problem solution for the unknown scattered field function $H_{z}^{s c}$ (Colton and Kress 1983). 
The resistive boundary condition is a well-established model of a thin penetrable material sheet. It is commonly expressed as the following pair of equations:

$$
\left[\vec{E}_{T}^{+}(\vec{r})+\vec{E}_{T}^{-}(\vec{r})\right]=2 \vec{R}(\vec{r}) \cdot \vec{n}(\vec{r}) \times\left[\vec{H}_{T}^{+}(\vec{r})-\vec{H}_{T}^{-}(\vec{r})\right], \quad \vec{E}_{T}^{+}(\vec{r})=\vec{E}_{T}^{-}(\vec{r}), \vec{r} \in M,
$$

where the subscript " $T$ " indicates the tangential field components, the superscripts " - " and "+" relate to the front and back faces of reflector, respectively, $\vec{r}$ is a vector on the $x-y$ plane, and the unit normal vector $\vec{n}$ is directed from the "-" to the "+" face.

As mentioned, the incident magnetic field is taken as the beam-like form generated by the CSP located at the complex-valued point $\vec{r}_{s}=\left(\vec{r}_{0}+i \vec{b}\right)=\left(x_{0}+i b \cos \beta, y_{0}+i b \sin \beta\right)$,

$$
H_{z}^{i n}(\vec{r})=H_{0}^{(1)}(k R), \quad R=\left|\vec{r}-\vec{r}_{s}\right|
$$

If $b \neq 0$, then this incident field function has two branch points which should be connected with a branch cut in the plane of real-valued coordinates. The center of the cut is at $\left(x_{0}, y_{0}\right)$ that is the real-space physical position of the CSP source. The absolute-value maximum of (3), at any distance from $\left(x_{0}, y_{0}\right)$, is found the along $\varphi=\beta$ direction in the polar coordinates having the origin at $\left(x_{0}, y_{0}\right)$. Therefore the parameters $b$ and $\beta$ can be conveniently imagined as the aperture width and the beam aiming angle, respectively, of a virtual aperture source. Note that the position point of the CSP feed is complex-valued, so that $r_{s}=\sqrt{\left(x_{0}+i b \cos \beta\right)^{2}+\left(y_{0}+i b \sin \beta\right)^{2}}$, however only $\operatorname{Re}\left(r_{s}\right)>0$ branch should be chosen for the physically meaningful case. The distance in the argument of (3) is found using the cosine theorem to be $R=\sqrt{r^{2}+r_{s}^{2}-2 r r_{s} \cos \left(\varphi-\theta_{s}\right)}$, so that it is also complexvalued. If the feed is in the first, more distant from the reflector, geometrical focus on the $x$ axis $\left(\left(x_{0}<0, y_{0}=0\right)\right.$ and the observation point is in the second geometrical focus, nearer to the reflector, then $\beta=0$ and $\theta_{s}=\pi$.

Further, for the $\mathrm{H}$ polarization formulation we need the tangential component of the incident electric field on the reflector. This field component can be found as follows:

$$
\vec{E}_{T}^{i n}=\vec{\tau}\left(-\frac{i Z_{0}}{k}\right) \frac{\partial}{\partial n} H_{z}^{i n}(\vec{r})
$$

where $\vec{\tau}$ is unit tangential vector on the contour $C$, and $Z_{0}$ and $k$ the free-space impedance and wavenumber, respectively.

The scattered electric field can be written by using auxiliary vector potentials formulation as (see, for instance, Oğuzer et al. 2004),

$$
E_{T}^{s c}=\frac{i Z_{0}}{k} \frac{\partial}{\partial l} \int_{M} \frac{\partial}{\partial l^{\prime}} J_{t^{\prime}}\left(\vec{r}^{\prime}\right) G\left(\vec{r}, \vec{r}^{\prime}\right) d l^{\prime}+i k Z_{0} \int_{M} J_{t^{\prime}}\left(\vec{r}^{\prime}\right) \cos \left[\xi(\vec{r})-\xi\left(\vec{r}^{\prime}\right)\right] G\left(\vec{r}, \vec{r}^{\prime}\right) d l^{\prime}
$$

where the scalar Green's function is the first-kind Hankel function of the zero order satisfying the radiation condition; i.e. $G\left(\vec{r}(\varphi), \vec{r}^{\prime}\left(\varphi^{\prime}\right)\right)=(i / 4) H_{0}^{(1)}\left(k\left|\vec{r}(\varphi)-\vec{r}^{\prime}\left(\varphi^{\prime}\right)\right|\right)$ and the unknown current density is defined as

$$
J_{t}\left(\vec{r}^{\prime}\right)=\vec{n} \times\left[\vec{H}_{z}^{+}\left(\vec{r}^{\prime}\right)-\vec{H}_{z}^{-}\left(\vec{r}^{\prime}\right)\right]
$$


Then by imposing the boundary conditions given in (2), the electric field integral equation (EFIE) is derived as follows:

$$
\begin{aligned}
R(\vec{r}) J_{t}(\vec{r})= & -\frac{i Z_{0}}{k} \frac{\partial H_{z}^{i n}}{\partial n}+\frac{i Z_{0}}{k} \frac{\partial}{\partial l} \int_{M}\left[\frac{\partial}{\partial l^{\prime}} J_{t^{\prime}}\left(\vec{r}^{\prime}\right)\right] G\left(\vec{r}, \vec{r}^{\prime}\right) d l^{\prime} \\
& +i k Z_{0} \int_{M} J_{t^{\prime}}\left(\vec{r}^{\prime}\right) \cos \left[\xi(\vec{r})-\xi\left(\vec{r}^{\prime}\right)\right] G\left(\vec{r}, \vec{r}^{\prime}\right) d l^{\prime}
\end{aligned}
$$

The EFIE given in (7) is an integro-differential equation having Cauchy kernel that is a higher singularity then the logarithmic one. The reduction of the singularity from the hyper-singular type to Cauchy type is done, as visible, at the expense of introducing the derivative of the unknown current-density function. Note that the presence of the first term containing resistivity does not change this singularity, and EFIE (7) reduces to the perfectly conducting (PEC) case if $R$ goes to zero. Therefore a convergent MAR-based numerical solution can be built similarly to the PEC reflector surface case (Oğuzer et al. 2004), with additional terms proportional to $R$.

\section{Regularization and discretization}

Assume that the curve $M$ can be characterized with the aid of the parametric equations $x=x(\varphi)$ and $y=y(\varphi)$, with $0 \leq|\varphi| \leq \theta$, in terms of the polar angle, $\varphi$. Besides, define the differential lengths in the tangential direction at any point on $M$ as $\partial l=a \rho(\varphi) \partial \varphi$ and $\partial l^{\prime}=a \rho\left(\varphi^{\prime}\right) \partial \varphi^{\prime}$, respectively, where $\rho(\varphi)=r(\varphi) /[a \cos \gamma(\varphi)], \xi(\varphi)$ is the angle between the normal on $M$ and the $x$-direction, $\gamma(\varphi)$ is the angle between the normal and the radial direction, and $\mathrm{a}$ is the radius of the circular arc $\mathrm{S}$.

Then we extend the surface-current density function, $J_{t^{\prime}}$, with zero value to $S$ and denote it now as $\tilde{J}_{t^{\prime}}$. Then the following dual set of equations is obtained on two complementary parts of complete contour $C$ :

$$
\begin{gathered}
-4(k a) \rho(\varphi) R_{0}(\varphi) \tilde{J}_{t}(\varphi)=4 i \frac{\partial H_{z}^{i n}}{\partial n} a \rho(\varphi)+\frac{\partial}{\partial \varphi} \int_{0}^{2 \pi}\left[\frac{\partial}{\partial \varphi^{\prime}} \tilde{J}_{t^{\prime}}\left(\varphi^{\prime}\right)\right] H_{0}^{(1)}\left[k\left|\vec{r}(\varphi)-\vec{r}^{\prime}\left(\varphi^{\prime}\right)\right|\right] d \varphi^{\prime} \\
+(k a)^{2} \int_{0}^{2 \pi} \tilde{J}_{t^{\prime}}\left(\varphi^{\prime}\right) \cos \left[\xi(\varphi)-\xi\left(\varphi^{\prime}\right)\right] H_{0}^{(1)}\left[k\left|\vec{r}(\varphi)-\vec{r}^{\prime}\left(\varphi^{\prime}\right)\right|\right] \rho(\varphi) \rho\left(\varphi^{\prime}\right) d \varphi^{\prime}, \\
\quad 0 \leq \varphi \leq \theta, \\
\tilde{J}_{t}(\varphi)=0, \quad \theta<|\varphi| \leq \pi,
\end{gathered}
$$

where $R_{0}(\varphi)=R(\varphi) / Z_{0}$ is the normalized position-dependent resistivity of the curved reflector.

To discretize the equation set 8(a) and (b), we use a Galerkin procedure with a set of entire-domain expansion functions. Then firstly the surface current density $\tilde{J}_{t^{\prime}}$ can be written in terms of the FS coefficients $x_{n}$ as follows

$$
\tilde{J}_{t}\left(\varphi^{\prime}\right)=\sum_{n=-\infty}^{\infty} x_{n} e^{i n \varphi^{\prime}} \varphi^{\prime} \in C
$$


Similarly, the incident field function can be expanded into FS coefficients on the whole curved contour $C$ as

$$
a \rho(\varphi) \frac{\partial}{\partial n} H_{z}^{i n}\left[k\left|\vec{r}(\varphi)-\vec{r}_{s}\right|\right]=\sum_{n=-\infty}^{\infty} z_{n} e^{i n \varphi}
$$

where the coefficients are found as

$$
z_{n}=\frac{a}{2 \pi} \int_{0}^{2 \pi} \rho(\varphi) \frac{\partial}{\partial n} H_{z}^{i n}\left[k\left|\vec{r}(\varphi)-\vec{r}_{s}\right|\right] e^{-i n \varphi} d \varphi
$$

To make computations more economic, we follow the procedure of Oğuzer et al. (2004) and add and subtract, in the kernels of (8), the functions similar to the actual ones however appearing in the case of $M$ being a circular arc. Then

$$
\begin{aligned}
G\left(\varphi, \varphi^{\prime}\right)= & \cos \left[\xi(\varphi)-\xi\left(\varphi^{\prime}\right)\right] \rho(\varphi) \rho\left(\varphi^{\prime}\right) H_{0}^{(1)}\left[k\left|\vec{r}(\varphi)-\vec{r}^{\prime}\left(\varphi^{\prime}\right)\right|\right] \\
& -\rho^{2}(\varphi) H_{0}^{(1)}\left\{2 k a\left|\sin \left[\left(\varphi-\varphi^{\prime}\right) / 2\right]\right|\right\} \\
H\left(\varphi, \varphi^{\prime}\right)= & H_{0}^{(1)}\left[k\left|\vec{r}(\varphi)-\vec{r}^{\prime}\left(\varphi^{\prime}\right)\right|\right]-H_{0}^{(1)}\left\{2 k a\left|\sin \left[\left(\varphi-\varphi^{\prime}\right) / 2\right]\right|\right\}
\end{aligned}
$$

The functions $H\left(\varphi, \varphi^{\prime}\right)$ and $G\left(\varphi, \varphi^{\prime}\right)$ have continuous first derivatives, their second derivatives with respect to $\varphi$ and $\varphi^{\prime}$ have only logarithmic singularities and hence belong to $L_{2}$. Therefore their FS coefficients, respectively $h_{n m}$ and $g_{n m}$, decay as $O\left(|n|^{-1.5-\varepsilon}|m|^{-1.5-\varepsilon}\right)$ on the curve $C$, with $\varepsilon>0$. The computation of these coefficients is a massive task, which can be computationally expensive; however this can be efficiently done with the aid of the FFT algorithm.

On the other hand, the arbitrary resistivity function can be expanded into FS coefficients as

$$
\rho(\varphi) R_{0}(\varphi)=\sum_{s=-\infty}^{\infty} q_{s} e^{i s \varphi}
$$

If all the above defined FS expansions are substituted into Eqs. 8(a) and (b), the following dual series equations are obtained:

$$
\begin{aligned}
& \sum_{n=-\infty}^{\infty} x_{n}|n|^{2} J_{n}(k a) H_{n}^{(1)}(k a) e^{i n \varphi}+\sum_{n=-\infty}^{\infty} e^{i n \varphi} \sum_{p=-\infty}^{\infty} x_{p} p n h_{n,(-p)} \\
& -\frac{2(k a)}{\pi} \sum_{n=-\infty}^{\infty} e^{i n \varphi} \sum_{p=-\infty}^{\infty} x_{p} q_{n-p} \\
& -(k a)^{2} \sum_{n=-\infty}^{\infty} e^{i n \varphi} \sum_{p=-\infty}^{\infty} x_{p}\left[J_{p}(k a) H_{p}^{(1)}(k a) \rho_{n-p}+g_{n,(-p)}\right] \\
& =\frac{2 i}{\pi} \sum_{n=-\infty}^{\infty} z_{n} e^{i n \varphi}, \quad \varphi \in M, \\
& \sum_{n=-\infty}^{\infty} x_{n} e^{i n \varphi}=0, \quad \varphi \in S,
\end{aligned}
$$

where $\rho_{n}$ are the FS coefficients of the function $\rho^{2}(\varphi)$ defined for $0 \leq \varphi \leq 2 \pi$. 
The dual series equations given in the 15(a) and (b) are converted to a special canonical dual series form. Then this canonical dual series equation is treated using the RHP technique that is making the analytical inversion of the most singular part of the original operator (See Appendix). As a result of this treatment, the following infinite matrix equation of the second kind is obtained:

$$
(I+A) X=B
$$

where $I=\left\{\delta_{m n}\right\}_{m, n=-\infty}^{+\infty}, \quad A=\left\{A_{m n}\right\}_{m, n=-\infty}^{+\infty}, \quad B=\left\{B_{m}\right\}_{m, n=-\infty}^{+\infty}, \quad X=\left\{x_{n}\right\}_{n=-\infty}^{+\infty}$ $\delta_{m n}$ is the Kroeneker symbol,

$$
\begin{gathered}
A_{m n}=\Delta_{n} T_{m n}+i \pi \sum_{p=-\infty}^{\infty} T_{m p}\left[n p h_{p,(-n)}-(k a)^{2} J_{n}(k a) H_{n}^{(1)}(k a) \rho_{p-n}\right. \\
\left.-(k a)^{2} g_{p,(-n)}-2 i(k a) q_{p-n}\right] \\
B_{m}=-2 \sum_{p=-\infty}^{\infty} z_{p} T_{m p},
\end{gathered}
$$

Provided that the functions $R_{0}(\varphi)$ and $\rho^{2}(\varphi)$ are smooth enough so that their FS coefficients belong to $l^{2}$ class and taking account of the above mentioned behavior of $h_{p n}$ and $g_{p n}$, this enables one to prove that $\sum_{m, n=-\infty}^{\infty}\left|A_{m n}\right|^{2}<\infty$. By the similar treatment one can find that $\sum_{m=-\infty}^{+\infty}\left|B_{m}\right|^{2}<\infty$ provided that the branch-cut associated with the CSP aperture does not cross the reflector contour $M$. In this case the infinite matrix Eq. (16) is of the Fredholm second kind. Hence the Fredholm theorems guarantee the existence of the unique exact solution and also the convergence of the sequence of approximate numerical solutions obtained from truncated (16) with progressively larger sizes $N_{t r}$.

\section{Radiation characteristics}

The scattering characteristics of a resistive reflector illuminated by the H-polarized CSP feed are determined by the far-zone field radiation pattern. Note that thanks to the fact that CSP field satisfies the radiation condition (unlike a Gaussian-beam field) we can include its radiation pattern to the total field,

$$
H_{z}(\vec{r})=\left[\Phi_{i n}(\varphi)+\Phi_{s c}(\varphi)\right](2 / i \pi k r)^{1 / 2} e^{i k r},
$$

where

$$
\begin{gathered}
\Phi_{i n}(\varphi)=e^{-i k r_{0} \cos \left(\varphi-\varphi_{0}\right)} e^{k b \cos (\varphi-\beta)} \\
\Phi_{s c}(\varphi)=\frac{1}{4} \sum_{n=-\infty}^{\infty} x_{n} \int_{-\theta}^{\theta} \cos \left(\varphi^{\prime}-\gamma^{\prime}-\varphi\right) e^{i n \varphi^{\prime}-i k r^{\prime}\left(\varphi^{\prime}\right) \cos \left(\varphi-\varphi^{\prime}\right)} k \rho\left(\varphi^{\prime}\right) d \varphi^{\prime}
\end{gathered}
$$

where $r_{0}=\left(x_{0}^{2}+y_{0}^{2}\right)^{1 / 2}$. However, if a reflector is elliptic, than the main interest is connected to its focusing effect in the near zone, i.e. in our case in the second geometrical focus that is nearer to reflector. The incident field has been already defined previously by (3) and (4). The scattered field $H_{z}^{s c}$ is computed by using the following integral: 


$$
H_{z}^{s c}(x, y)=a \int_{-\theta}^{\theta} \tilde{J}_{t}\left(\varphi^{\prime}\right) \frac{\partial G}{\partial n^{\prime}} \rho\left(\varphi^{\prime}\right) d \varphi^{\prime}
$$

where

$$
\frac{\partial G}{\partial n^{\prime}}=\frac{i k}{4} \frac{H_{1}^{(1)}(k R)}{a \rho\left(\varphi^{\prime}\right) R}\left\{-\left[x-x\left(\varphi^{\prime}\right)\right] \frac{d y\left(\varphi^{\prime}\right)}{d \varphi^{\prime}}+\left[y-y\left(\varphi^{\prime}\right)\right] \frac{d x\left(\varphi^{\prime}\right)}{d \varphi^{\prime}}\right\}
$$

and $R^{2}=\left[x-x\left(\varphi^{\prime}\right)\right]^{2}+\left[y-y\left(\varphi^{\prime}\right)\right]^{2}$.

Here, the current $\tilde{J}_{t}\left(\varphi^{\prime}\right)$ is expanded in terms of FS defined in Eq. (9) where the coefficients $x_{n}$ are obtained by the solution of the matrix Eq. (16). Besides, $x\left(\varphi^{\prime}\right)$ and $y\left(\varphi^{\prime}\right)$ are the coordinates of the source point on the reflector surface and $x$ and $y$ are the coordinates of the observation point. In the presented below numerical results, we have computed the magnetic field in the second geometrical focus and normalized the total field magnitude value by the incident field value at the middle point of the CSP beam aperture.

The total power radiated by the CSP feed in the presence of reflector is

$$
P_{\text {rad }}(R)=\frac{Z_{o}}{\pi k} \int_{0}^{2 \pi}\left|\Phi_{i n}(\varphi)+\Phi_{s c}(\varphi)\right|^{2} d \varphi
$$

The total radiated power is a function of the reflector resistivity $R$ as well as the source and reflector geometry parameters. Together with the absorbed power captured by the imperfect reflector surface, must satisfy the power conservation law,

$$
P_{s p l}=P_{r a d}+P_{a b s},
$$

where $P_{s p l}$ is the power supplied by the source in the given environment. This quantity in the case of CSP beam cannot be reduced to the total field value in the source point as it is done with conventional line-current source. Instead, it can be converted to the integration around arbitrary circular contour enclosing the CSP. It is convenient to choose an auxiliary coordinate system located on the feed position i.e. the first focus point and a circle with radius $a_{f}$ is assumed around this origin point $O_{f}$ (see Fig. 1). The radius $a_{f}$ can be any value unless it does not reach to the reflector surface. Then the scattered electric and magnetic fields from the reflector are sampled on this new circle depending on the angle parameter $\varphi_{f}$. The required FS coefficients are computed by the available FFT algorithm. The total fields on the defined circle can be represented as

$$
\begin{aligned}
& H_{z}^{T}\left(\varphi_{f}\right)=\sum_{p=-\infty}^{\infty}\left(h_{p}^{i n}+h_{p}^{s c}\right) e^{i p \varphi_{f}} \\
& E_{\varphi_{f}}^{T}\left(\varphi_{f}\right)=\sum_{p=-\infty}^{\infty}\left(e_{p}^{i n}+e_{p}^{s c}\right) e^{i p \varphi_{f}}
\end{aligned}
$$

where $h_{p}^{s c}$ and $e_{p}^{s c}$ are the above mentioned FS coefficients of the scattered fields on the defined circle. The incident fields can be expressed easily on the mentioned circle and so their FS coefficients are given as $h_{p}^{i n}=(i / 4) J_{p}(i k b) H_{p}^{(1)}\left(k a_{f}\right)$ and $e_{p}^{i n}=\left(Z_{0} / 4\right) J_{p}(i k b) H_{p}^{(1)^{\prime}}\left(k a_{f}\right)$. The total time average real power supplied by the feed antenna in the presence of the reflector 
surface i.e. the input power is obtained by integrating the power flow density over the defined circle,

$$
P_{s p l}=\frac{1}{2} \int_{0}^{2 \pi} \operatorname{Re}\left(E_{\varphi_{f}}^{T} H_{z}^{T *}\right) a_{f} d \varphi_{f}
$$

Then the supplied power can be expressed as follow

$$
P_{s p l}=\pi a_{f} \sum_{p=-\infty}^{\infty} \operatorname{Re}\left[\left(e_{p}^{i n}+e_{p}^{s c}\right)\left(h_{p}^{i n}+h_{p}^{s c}\right)^{*}\right]
$$

This quantity depends indirectly on the FS coefficients $x_{n}$ of the surface current density induced on the reflector surface. These coefficients are included into the $e_{p}^{s c}$ and $h_{p}^{s c}$ parameters which represent the fields scattered from the reflector. Also it is verified that the variation of the radius $a_{f}$ does not make any change in the supplied power $P_{s p l}$.

In computations, the power values explained above are normalized by the power radiated by the same CSP source into free space,

$$
P_{0}=(2 / k) Z_{0} I_{0}(2 k b)
$$

\section{Numerical results}

The presented formulation has been verified using various characteristics of the elliptic reflector illuminated by a CSP source as shown in Fig. 1, i.e. with $\varphi_{0}=\pi$. For the sake of performance comparison, we have also applied the method of moments (MoM) to the same problem. Here we have used Galerkin approach choosing the triangular subdomain basis and testing functions. The main matrix in the MoM formulation contains the double integrals which have been computed using the optimized routines of Matlab 6.9. To generate all these numerical results we have used a desktop PC core i-5 computer with 4GB RAM and Windows 7 operating system.

In the computation of the Fourier coefficients of the defined functions $g_{n m}$ and $h_{n m}$, we used Fast Fourier Transform (FFT) algorithm with 2048-points taken. The FFT approach enables us to solve electrically large reflectors within a reasonable time. To check the accuracy and convergence for the surface current density i.e. in the near field computations, we used the following norm expression for the relative error: $\varepsilon_{c u r}\left(N_{t r}\right)=\max \left|x_{n}^{N_{t r}}-x_{n}^{N_{t r}+1}\right|$. $\left(\max \left|x_{n}^{N_{t r}}\right|\right)^{-1}$ where $x_{n}^{N_{t r}}$ is the FS coefficients of the surface current density obtained from the matrix equation truncated to $N_{t r}$. For example, in Fig. 2 we present the variation of the relative error with the truncation number in the determination of the surface current density for uniformly resistive lossy reflectors. As seen in Fig. 2a, the lower resistivity case converges faster than the higher one. This is the opposite behavior to the case of E-polarization and similar to the results obtained for a circular uniform-resistivity reflector under the H-polarized illumination (Nosich et al. 1997).

In Fig. 2b, we see that making the reflector size larger leads to the necessity of working with larger matrices but do not slow the convergence rate. This is also an expected result because a larger aperture dimension requires more terms in (9) to approximate the surface current (the same takes place for a PEC reflector).

In Fig. 3, the relative error in the normalized field amplitude at the second focus of the elliptic reflector antenna is plotted as a function of $N_{t r}$. In Fig. 3a, b, two different results are 
Fig. 2 The relative error in the surface current density versus the matrix truncation number, $\mathrm{Ntr}$, for the uniformly resistive lossy reflectors with $\mathrm{e}=0.5$ (elliptic) and $\mathrm{kb}=3$. a Solid line is for $\mathrm{R}=0.2 Z_{0}$ and dashed line is for $\mathrm{R}=Z_{0}$, with other parameters being $\mathrm{d}=20 \lambda$ and $\mathrm{f} / \mathrm{d}=0.5$. b Solid line is for $\mathrm{d}=20 \lambda$ and dashed line is for $\mathrm{d}=40 \lambda$, with other parameters being $\mathrm{f} / \mathrm{d}=0.5$ and $\mathrm{R}=Z_{0}$
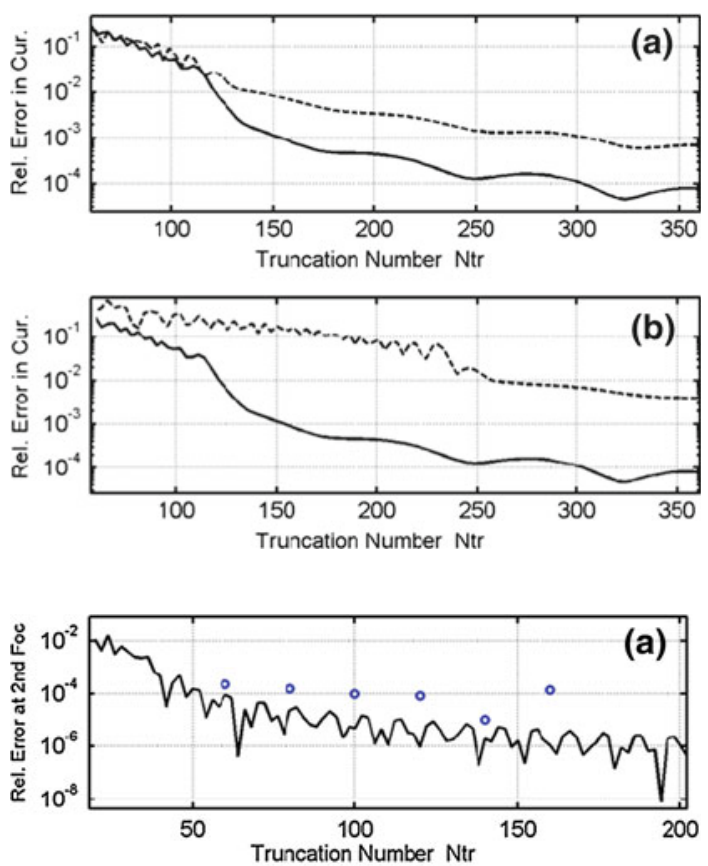

Fig. 3 The relative errors in the ratio of the field amplitude at the second focus to the incident field at the middle aperture versus the matrix truncation number, Ntr, computed with MoM and our method for the uniformly resistive lossy ( $\mathrm{R}$ is real-valued) and elliptic reflectors with $\mathrm{d}=10 \lambda, \mathrm{f} / \mathrm{d}=0.4, \mathrm{~kb}=3$ and $\mathrm{e}=0.5$. a $\mathrm{R}=0.1 Z_{0}$

b $\mathrm{R}=0.7 Z_{0}$. In both cases solid line MAR and circle points indicate the MoM results

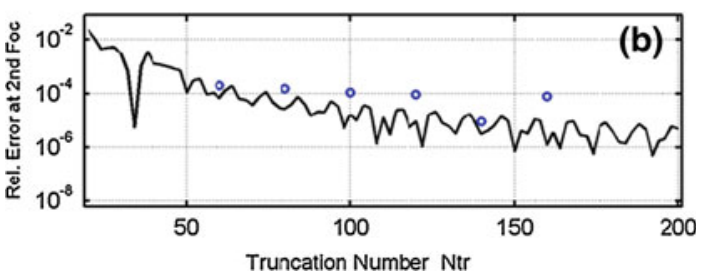

given for a lower and a higher lossy resistivity cases and comparison is presented between the MoM and MAR data. As expected, our MAR-based results have better convergence rate than MoM, and there is a 1-2 digit difference in accuracy. Also it should be said that our MoM code works much slower than the MAR algorithm. This is because the reflector contour is not a circle and the double integral appearing in the MoM formulation cannot be reduced to a single integral. Still if the observation point moves away from the reflector (i.e. from the current location) to the near field (such as the second focus), the relative accuracy in the computation gets improved by 1 or 2 digits.

In the further figures, we assume that the CSP feed is located in the first focus of the elliptic reflector (further from the reflector) and observe the field amplitude at the second focus (nearer to the reflector). This will provide us an understanding of the performance of the elliptic-reflector focuser.

Figure 4 shows the variation of the normalized magnetic field amplitude in the second focus with the $f / d$ ratio with a fixed $d$ value (i.e. for a fixed reflector aperture dimension but a varying depth). Two different curves are obtained for two different $d$ values. For both of them, the curves oscillate and show a gradual decrement, apparently because the distance between the feed and observation point gradually increases for larger $f / d$. Besides, it should be said that the envelope of the oscillatory curves is higher for the lower aperture dimension 


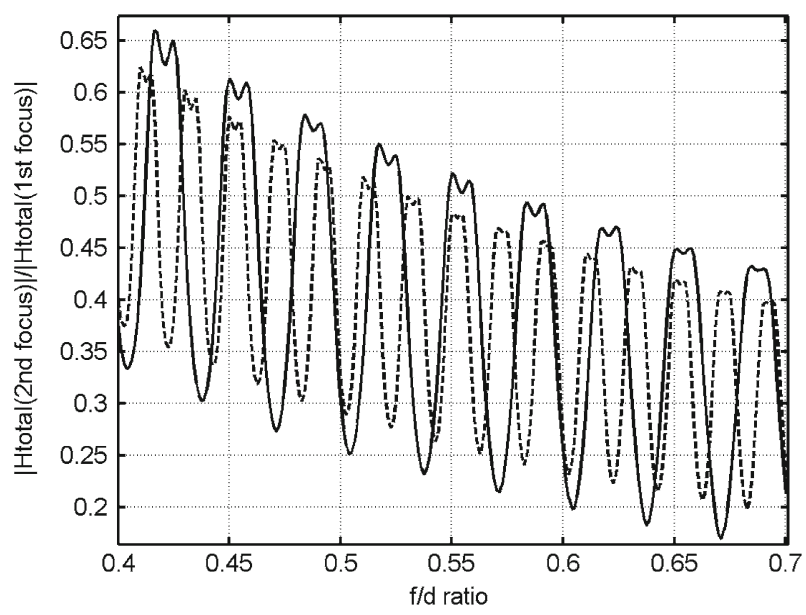

Fig. 4 The ratio of the $\mathrm{H}$ field amplitude at the second focus to the incident field at the middle aperture varies with $\mathrm{f} / \mathrm{d}$ under the fixed aperture length $\mathrm{d}$. The solid line is for $\mathrm{d}=15 \lambda$ and the dashed line is for $\mathrm{d}=25 \lambda$. Here, the other problem parameters are given as $\mathrm{kb}=3, \mathrm{R}=0.4 \mathrm{Zo}$ and $\mathrm{e}=0.5$

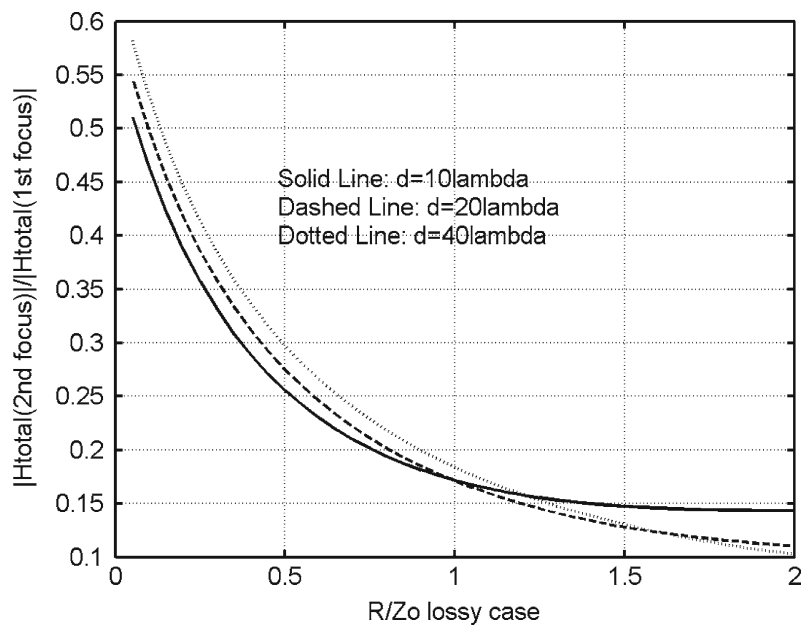

Fig. 5 The ratio of the $\mathrm{H}$ field amplitude at the second focus to the incident field at the middle aperture varies with $\mathrm{R} / \mathrm{Zo}$ under the fixed aperture length $\mathrm{d}$ for various $\mathrm{d}$ values. The solid line is for $\mathrm{d}=10 \lambda$, the dashed line is for $\mathrm{d}=20 \lambda$ and the dotted line is for $\mathrm{d}=30 \lambda$. Here, the other problem parameters are given as $\mathrm{kb}=3$, $\mathrm{f} / \mathrm{d}=0.5$ and $\mathrm{e}=0.5$ (elliptic). (only lossy case)

$d$ as expected due to the lower distance between the source and reflector and so it produces higher scattering field value at the second focus point.

Figure 5 shows the variation of the normalized magnetic field amplitude in the second focus as a function of the surface resistivity (lossy case). It is seen that the higher-resistivity surface provides less efficient focusing that is clearly because of power leakage through the more transparent reflector surface. Still larger reflectors provide better focusing than smaller ones. But the situation is completely different for higher resistivity cases. For this case, the lower aperture dimension produces the better focusing at the second focus of the elliptic reflector. 


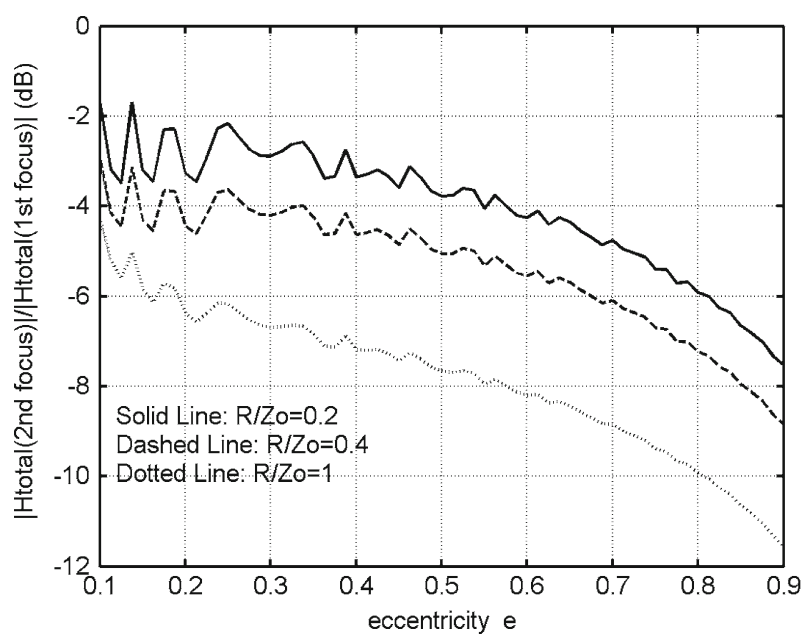

Fig. 6 The ratio of the $\mathrm{H}$ field amplitude at the second focus to the incident field at the middle aperture varies with eccentricity parameter of the ellipse " $e$ " under the fixed surface resisitivity R/Zo (lossy case) for various $\mathrm{R} / \mathrm{Zo}$ values. The solid line is for $\mathrm{R} / \mathrm{Zo}=0.2$, the dashed line is for $\mathrm{R} / \mathrm{Zo}=0.4$ and the dotted line is for $\mathrm{R} / \mathrm{Zo}=1$. Here, the other problem parameters are given as $\mathrm{kb}=3, \mathrm{~d}=20 \lambda, \mathrm{Ntr}=200$ and $\mathrm{f}=10 \lambda$

Fig. 7 The ratio of the $\mathrm{H}$ field amplitude at the second focus to the incident field at the middle aperture varies with the aperture length $d / \lambda$ under the fixed feed illumination $\mathrm{kb}$ for various $\mathrm{kb}$ values. The solid line is for $\mathrm{kb}=3$, the dashed line is for $\mathrm{kb}=5$ and the dotted line is for $\mathrm{kb}=7$. a $\mathrm{R} / \mathrm{Zo}=0.4$ case. $\mathbf{b} \mathrm{R} / \mathrm{Zo}=0.8$ uniform lossy resistive case. Here, the other problem parameters are given as $\mathrm{f} / \mathrm{d}=0.5$, $\mathrm{Ntr}=350$ and $\mathrm{e}=0.4$ (elliptic case)
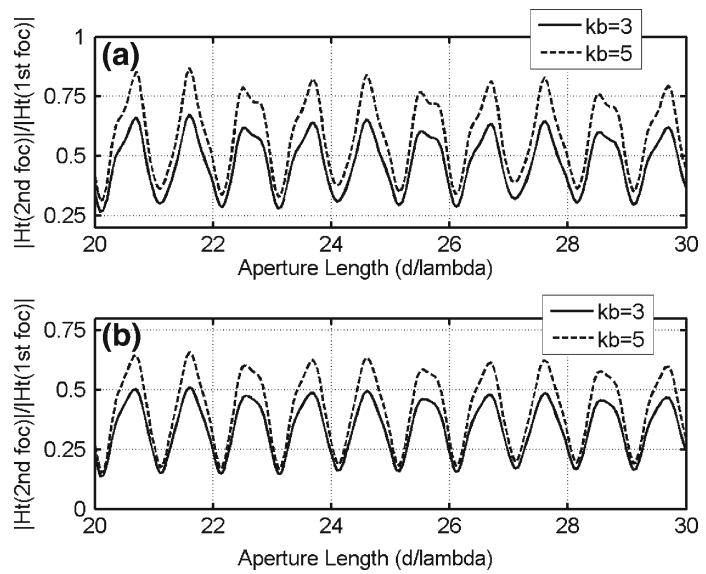

In Fig. 6 the field amplitude in the second focus varies with the eccentricity value $e$ of the elliptic reflector surface. These variations are obtained for three different cases of the normalized resistivities $R / Z_{0}$. It is seen that the high field amplitude is obtained at the second focus for the lower resistivity (close to PEC) case. Also all three curves go down if the eccentricity $e$ increases. This is due to the greater distance between the source and reflector that leads to the less intensive scattering from the reflector surface.

Figure 7 presents the variation of the field amplitude in the second focus with the increasing aperture dimension $d$ of the reflector at the fixed $f / d$ ratio. The plots are obtained for two different $R / Z_{0}$ values in (a) and (b). As one can see, the part (b) curves are lower than the corresponding part (a) ones. This is expected result because the higher-resistivity reflector in 
Fig. 8 Absorbed and radiated power variations with the R/Zo lossy surface resistivity under the fixed f/d ratio for various f/d values. The aperture length is fixed and given as $\mathrm{d}=30 \lambda$. Solid line is for $\mathrm{f} / \mathrm{d}=0.4$ and $\mathrm{f}=12 \lambda$, dashed line is for $\mathrm{f} / \mathrm{d}=0.5$ and $\mathrm{f}=15 \lambda$, dotted line is for $\mathrm{f} / \mathrm{d}=0.6$ and $\mathrm{f}=18 \lambda$. a Absorbed power variation $\mathbf{b}$ Radiated power variation. Here, the other problem parameters are given as $\mathrm{kb}=3$, $\mathrm{e}=0.4$ (ellipse) and $\mathrm{Ntr}=300$
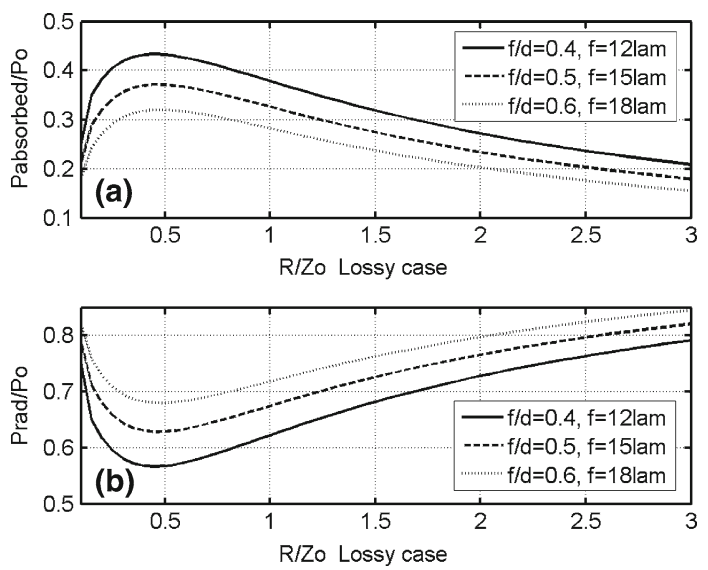

(b) is more transparent than the lower-resistivity one in (a). The increasing distance $d$ also increases the focus distance $\mathrm{f}$ under the fixed $f / d$ ratio. Then due to the varying distance, the interference produces the curves presented in Fig. 7a, b. In the same figure, two different edge illumination cases are presented. It is seen that the more directive feed illumination produces higher focusing effect. In this case the edges are illuminated weaker and the feed radiation bypassing the reflector is smaller, so more power is reflected from the reflector surface and gathers in the second focus point.

Figure 8 shows the variation of the normalized power absorbed by the reflector and the normalized total power radiated from the same system. One can see that for the variation of the focal distance $f$ with the fixed $d$ value as $30 \lambda$, the $f / d$ ratio also varies. This does not change the elliptic surface eccentricity $e$ and hence the feed only becomes closer to the reflector if the focal distance gets smaller. Therefore in both parts of the same figure for the lower focus distances the absorbed power is larger because the field value on the resistive surface becomes higher in this case. The absorbed power curves reach peak values and then drops to smaller values gradually. Finally one can observe that the absorbed and radiated powers as a function of resistivity vary oppositely to each other. Together they are very close to unity that means that the power supplied by the CSP feed in the presence of imperfect resistive reflector remains close to its free-space counterpart. This is a result of non-resonant behavior of the reflector.

\section{Conclusions}

In this study, the H-polarized wave scattering from the arbitrary-profile 2-D reflector with varying resistivity has been analyzed using the SIE, dual series equations and the analytical regularization technique. Therefore the obtained results can be considered as accurate data for the validation of the other numerical approximations. As an example of implementation, we have studied the re-focusing of the complex-source-point beam from one focus of the elliptic reflector to the other. These results provide the information necessary for a better understating of various optical systems like elliptic focusers of laser pumping circuits. They demonstrate the importance of the proper choice of the edge illumination and show the effect of the partial transparency of reflector on the focusing ability. 


\section{Appendix}

The canonical dual series equation described in Oğuzer et al. (2004) can be solved by the application of the RHP technique (Nosich 1993), and the resultant solution can be given as

$$
x_{m}=\sum_{n=-\infty}^{\infty} f_{n} T_{m n}(\theta),
$$

where the coefficients $T_{m n}(\theta)$ depend on elementary functions as given by the formulas presented in Nosich (1993), Oğuzer et al. (1995) and do not need numerical integration. Note that in our case the right hand part FS coefficients defined as $f_{n}$ are given as follows:

$$
\begin{aligned}
f_{n}= & -x_{n} \Delta_{n}-i \pi \sum_{p=-\infty}^{\infty} x_{p}\left[p n h_{n,(-p)}-(k a)^{2} J_{p}(k a) H_{p}^{(1)}(k a) \rho_{n-p}\right. \\
& \left.-(k a)^{2} g_{n,(-p)}-2 i(k a) q_{n-p}\right]-2 z_{n}
\end{aligned}
$$

where $\Delta_{n}=i \pi|n|^{2} J_{n}(k a) H_{n}^{(1)}(k a)-|n|$. The large-index asymptotics for cylindrical functions show that if $n \rightarrow \infty$, then $\Delta_{n}=O\left(n^{-1}\right)$. Then the FS coefficients given in Eq. (32) are substituted into the Eq. (31) and, after the change of the summation orders, we obtain the resultant matrix equation.

\section{References}

Anastassiu, H., Pathak, P.: High-frequency analysis of Gaussian beam scattering by a two-dimensional parabolic contour of finite width. Radio Sci. 30, 493-503 (1995)

Barclay, M.R., Rusch, W.V.T.: Moment-method analysis of large, axially symmetric reflector antennas using entire domain functions. IEEE Trans. Antennas Propag. 39, 491-496 (1991)

Boriskina, S.V., Nosich, A.I., Altintas, A.: Effect of the imperfect flat earth on the vertically-polarized radiation of a cylindrical reflector antenna. IEEE Trans. Antennas Propag. 48, 285-292 (2000)

Chou, H.T., Pathak, P.H., Burkholder, R.J.: Application of Gaussian-ray basis functions for the rapid analysis of electromagnetic radiation from reflector antennas. Proc. Inst. Electr. Eng. 150, 177-183 (2003)

Colton, D., Kress, R.: Integral Equation Method in Scattering Theory. Wiley, Berlin (1983)

Goldsmith, P.F.: Quasioptical Systems: Gaussian Beams, Quasioptical Propagation and Applications. IEEE Press, NY (1998)

Hasselmann, F.J.V., Felsen, L.B.: Asymptotic analysis of parabolic reflector antennas. IEEE Trans. Antennas Propag. 30, 677-685 (1982)

Heldring, A., Rius, J.M., Ligthart, L.P., Cardama, A.: Accurate numerical modeling of the TARA reflector system. IEEE Trans. Antennas Propag. 52, 1758-1766 (2004)

Hower, G.L., Olsen, R.G., Earls, J.D., Scheider, J.B.: Inaccuracies in numerical calculation of scattering near to natural frequencies. IEEE Trans. Antennas Propagat. 41, 982-986 (1993)

Idemen, M., Büyükaksoy, A.: High frequency surface currents induced on a perfectly conducting cylindrical reflector. IEEE Trans. Antennas Propag. 32, 501-507 (1984)

Jenn, D.C., Morgan, M.A., Pogorzelski, R.J.: Characteristics of approximate numerical modeling techniques applied to resonance-sized reflectors. Electromagnetics 15, 41-53 (1995)

Martinez-Burdalo, M., Martin, A., Villar, R.: Uniform PO and PTD solution for calculating plane wave backscattering from a finite cylindrical shell of arbitrary cross section. IEEE Trans. Antennas Propag. 41, 1336-1339 (1993)

Mittra, R., Lee, S.W.: Analytical Techniques in the Theory of Guided Waves. Macmillan Publ, New York (1971)

Nosich, A.I.: Green's function-dual series approach in wave scattering from combined resonant scatterers. In: Hashimoto, M., Idemen, M., Tretyakov, O.A. (eds.) Analytical and Numerical Methods in Electromagnetic Wave Theory, pp. 419-469. Science House, Tokyo (1993)

Nosich, A.I., Okuno, Y., Shiraishi, T.: Scattering and absorption of E and H-polarized plane waves by a circularly curved resistive strip. Radio Sci. 31, 1733-1742 (1996) 
Nosich, A.I., Yurchenko, V.B., Altintas, A.: Numerically exact analysis of a two-dimensional variableresistivity reflector fed by a complex point source. IEEE Trans. Antennas Propag. 45, 1592-1601 (1997)

Nosich, A.I.: Method of analytical regularization in the wave-scattering and eigenvalue problems: foundations and review of solutions. IEEE Antennas Propag. Mag. 42, 34-49 (1999)

Oğuzer, T., Altintas, A., Nosich, A.I.: Accurate simulation of reflector antennas by the complex source-dual series approach. IEEE Trans. Antennas Propag. 43, 793-802 (1995)

Oğuzer, T.: Analysis of circular reflector antenna covered by concentric dielectric radome. IEEE Trans. Antennas Propag. 49, 458-463 (2001)

Oğuzer, T., Nosich, A.I., Altıntas, A.: E-Polarized beam scattering by an open cylindrical PEC strip having arbitrary conical-section profile. Microw. Opt.Techn. Lett. 31, 480-484 (2001)

Oğuzer, T., Nosich, A.I., Altıntaş, A.: Analysis of an arbitrary profile cylindrical reflector antenna, H-polarization case. IEEE Trans. Antennas Propag. 52, 3156-3162 (2004)

Oğuzer, T., Altıntaş, A.: Analysis of the 2D nonconcentric reflector antenna-in-radome system: E-Polarization case. J. Electromagn. Waves Appl. 19, 2093-2111 (2005)

Oğuzer, T., Altıntaş, A.: Analysis of the nonconcentric reflector antenna in radome system by the iterative reflector antenna and radome interaction. J. Electromagn. Waves Appl. 21, 57-70 (2007)

Oğuzer, T., Altintas, A., Nosich, A.I.: Integral equation analysis of an arbitrary-profile and varying-resistivity cylindrical reflector illuminated by an E-polarized complex-source-point beam. J. Opt. Soc. Am. A. 26, 1525-1532 (2009)

Rieckmann, C.: Novel modular approach based on Gaussian beam diffraction for analyzing quasi-optical multireflector antennas. Proc. Inst. Elect. Eng. Microw. 149, 160-167 (2002)

Scott, C.: Modern Methods of Reflector Antenna Analysis and Design. Artech House, Norwood (1990)

Senior, T.B.A.: Some problems involving imperfect half-planes. In: Uslenghi, P.L.E. (ed.) Electromagnetic Scattering, pp. 185-219. Academic, London (1978)

Suedan, G.A., Jull, E.V.: Beam diffraction by planar and parabolic reflectors. IEEE Trans. Antennas Propag. 39, 521-527 (1991)

Thumm, M.K., Kasparek, W.: Passive high-power microwave components. IEEE Trans. Plasma Sci. 30, 755786 (2002)

Umul, Y.Z.: Scattering of a Gaussian beam by an impedance half-plane. J. Opt. Soc. Am. A. 24, 3159-3167 (2007)

Umul, Y.Z.: Scattering of a line source by a cylindrical parabolic impedance surface. J. Opt. Soc. Am. A. 25, 1652-1659 (2008)

Yurchenko, V.B., Altintas, A., Nosich, A.I.: Numerical optimization of a cylindrical reflector-in-radome antenna system. IEEE Trans. Antennas Propag. 47, 668-673 (1999) 